\title{
POLYSILICON STRUCTURES FOR SHEAR STRESS SENSORS
}

\author{
Fukang Jiang, Yu-Chong Tai \\ Department of Electrical Engineering \\ California Institute of Technology \\ Pasadena, CA 91125, USA \\ Jin-Biao Huang, Chih-Ming Ho \\ Department of Mechanical, Aerospace and Nuclear Engineering \\ University of Caiifornia \\ Los Angeles, CA 90024, USA
}

\begin{abstract}
Four types of micromachined polysilicon structures have been designed and fabricated for wall shear stress sensors in flow measurement and control. Their frequency responses, heat transfer characteristics and windtunnel responses have been extensively studied. They are all useful but one may be better than the others depending on the application requirement.
\end{abstract}

\section{INTRODUCTION}

Recently, much effort has been made to control the turbulent boundary layer flows and to reduce the drag of a moving object [1-3]. For this, fully understanding the wall turbulence is very important. Moreover, the development of adequate devices - sensors and actuators - to realize the control is also challenging. Among various methods used in turbulence detection, the measurement of near wall shear stress gives the most useful information, although it is more difficult. There are many ways to measure the wall shear stress [4]. Among them, the thermal method, which uses hot film sensors to determine shear stress indirectly, has many advantages over other techniques for real time flow control.

The traditional hot film sensors are thin metal film resistors deposited on substrates. Since heat convection is related to the change of shear stress, it is desirable to thermally isolate the thin film resistor from the substrate to minimize the conductive heat loss. In the past, the only way to partially solve the problem was to use low thermal conductivity materials such as quartz for the substrates. Here, it is demonstrated that improvements can be made in both materials and structures if silicon surface micromachining technology is used. Here, we report our experimental study on four types of microfabricated polysilicon shear stress sensors.

\section{DESIGN AND FABRICATION}

Fig. 1 shows the cross sections of these four types of

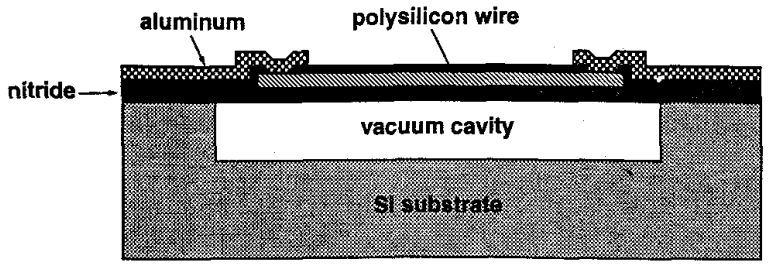

(a) Type I: Hot film.

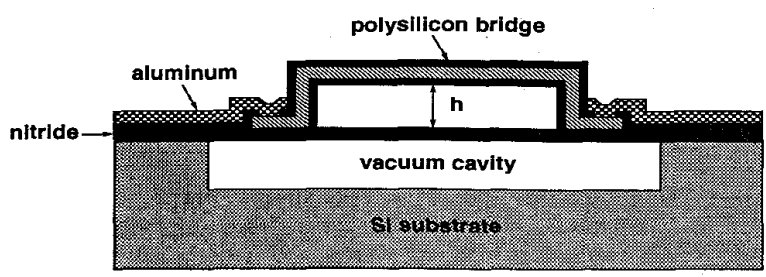

(b) Type II: Bridge over vacuum cavity.

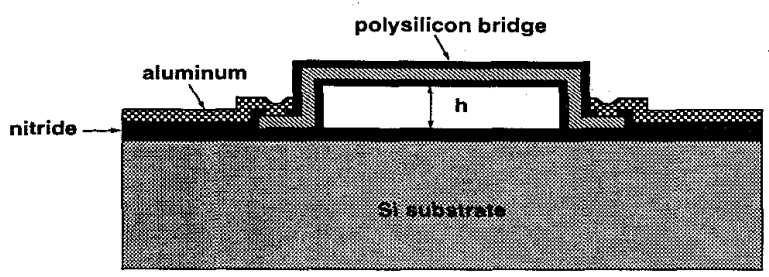

(c) Type III: Bridge over substrate.

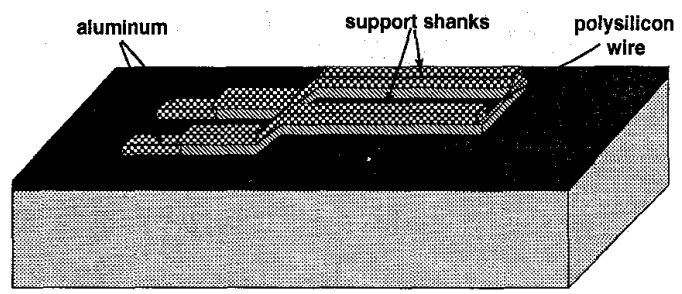

(d) Type IV: Hot wire.

Fig. 1 Structures of the sensors

sensors. Type I features a $2 \mu \mathrm{m}$ deep vacuum cavity with a $0.25 \mu \mathrm{m}$ thick polysilicon wire embedded in the nitride diaphragm. Here the vacuum cavity is designed for good thermal isolation of the diaphragm from the substrate [5]. 

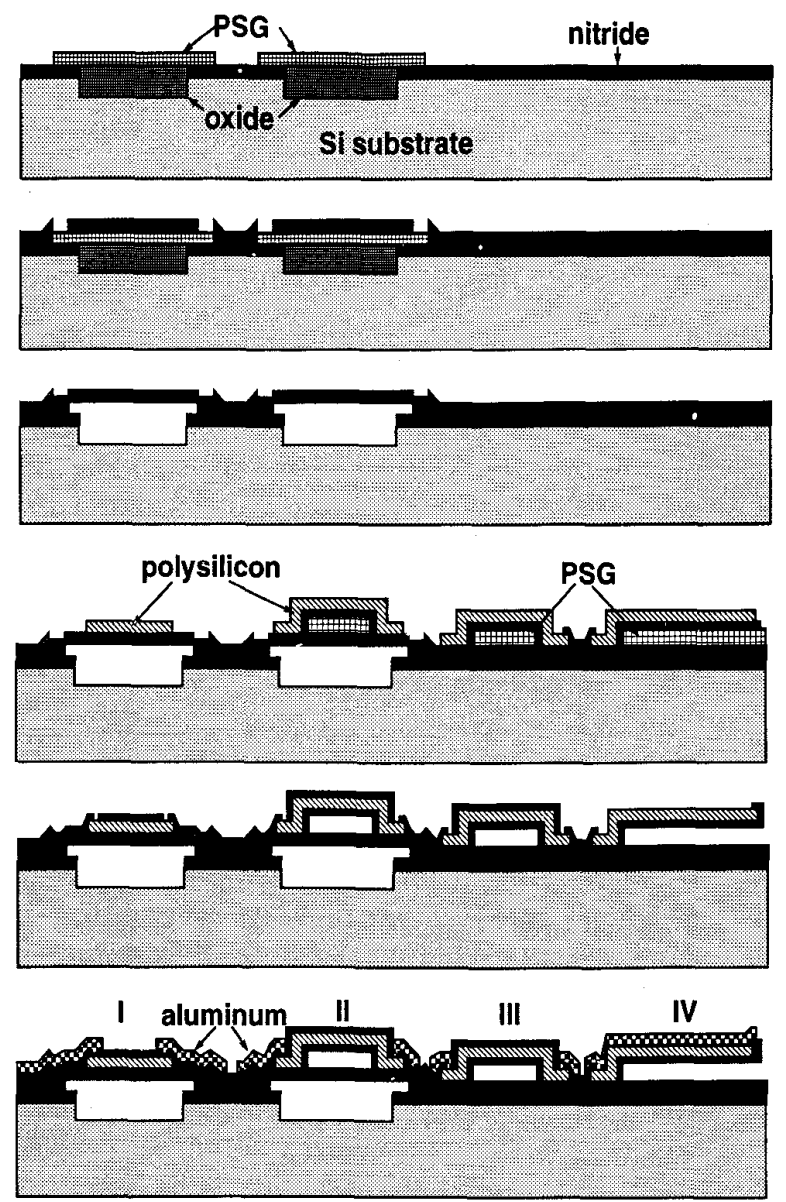

Fig. 2 Fabrication process flow.

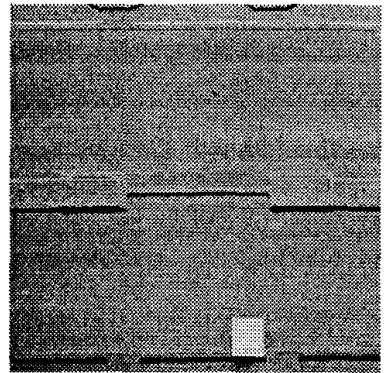

(a) Type I

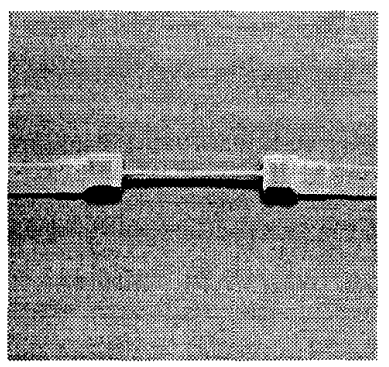

(c) Type III

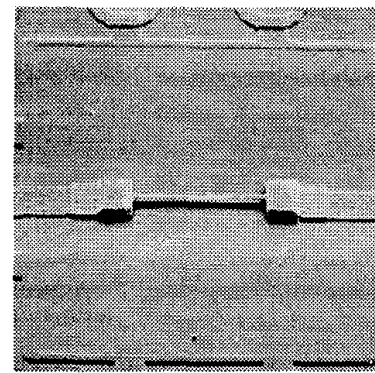

(b) Type II

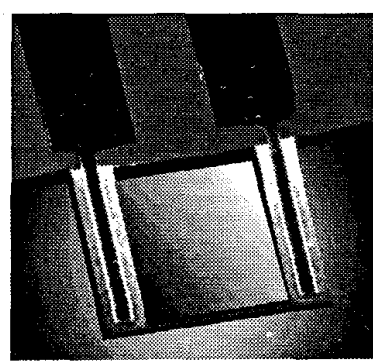

(d) Type IV
Fig. 3 SEM photographs of the sensors.
Type II has a similar structure to type I except that the polysilicon wire is lifted $1-5 \mu \mathrm{m}$ above the diaphragm, thus achieving better thermal isolation. Type III is a conventional polysilicon bridge sitting on the solid substrate [6]. Type IV is basically a micromachined hot wire close to a wall as has been previously reported. The wire is a few microns above the substrate surface and is in the linear velocity distribution region so that it measures the wall shear stress instead of velocity [7-8]. All four types were fabricated on a single chip using the process shown in Fig. 2 to ensure identical thermal and electrical properties of the sensor materials.

The fabrication starts with the deposition and patterning of $400 \mathrm{~nm}$ LPCVD low stress silicon nitride on 4-inch (100) $\mathrm{Si}$ wafers to define the cavities. The windows are etched down $700 \mathrm{~nm}$ using plasma etching. The subsequent high temperature oxidation planarizes the windows with the growth of $1.6 \mu \mathrm{m}$ thick silicon dioxide. A $400 \mathrm{~nm}$ thick LPCVD PSG layer is then deposited and patterned, followed by the deposition of $1 \mu \mathrm{m}$ low stress nitride as the diaphragm. Small holes are opened in the nitride to expose the PSG. Next, $49 \%$ HF is used to etch away the PSG and the thermal oxide in the cavities. After the wafer is spindried, a $600 \mathrm{~nm}$ LTO and $400 \mathrm{~nm}$ nitride composite layer is deposited and patterned to seal the etch holes. Another layer of thick PSG between 1-5 $\mu \mathrm{m}$ is then deposited and patterned to form the sacrificial spacer between the polysilicon bridge and the substrate (or the diaphragm), followed by annealing at $1100^{\circ} \mathrm{C}$ to reflow the PSG and round the edges. Next, $200 \mathrm{~nm}$ of nitride and $250 \mathrm{~nm}$ of polysilicon are deposited. The polysilicon is doped by $40 \mathrm{keV}$ boron ion implantation with a dose of $1 \times 10^{16} / \mathrm{cm}^{3}$. After annealing at $1000^{\circ} \mathrm{C}$ to activate the dopants, another 200 $\mathrm{nm}$ of nitride is deposited. The two layers of nitride are patterned in one step to encapsulate the polysilicon wire. Again, $49 \% \mathrm{HF}$ is used to etch the PSG spacers to release the polysilicon bridges. After rinsing in DI water, the wafer is dipped in alcohol and then baked to prevent the stiction of the bridge to substrate. It turns out that the $200 \mathrm{~nm}$ nitride underneath the polysilicon also helps to ease the stiction problem. Finally, $800 \mathrm{~nm}$ aluminum is

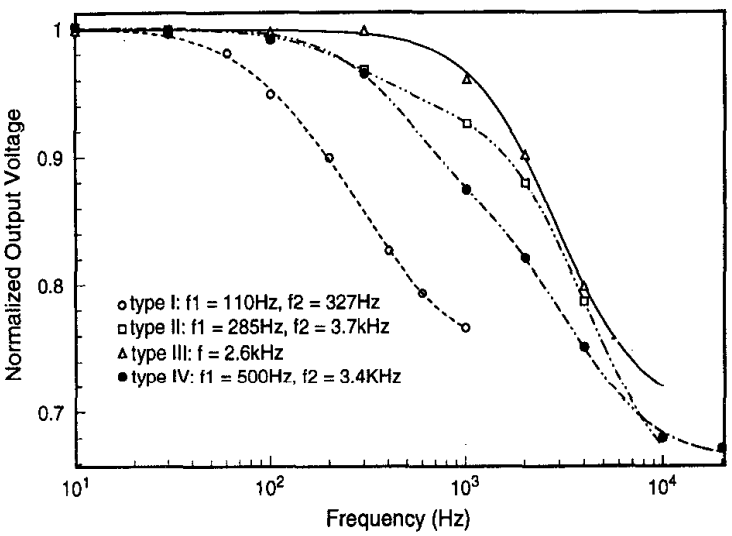

Fig. 4 Frequency responses in constant current mode. 
evaporated and patterned to finish the whole process. Fig. 3 shows the SEM photographs of the $3 \mu \mathrm{m}$ wide and 120 $\mu \mathrm{m}$ long sensors. All the following measurement results shown in this paper are for the sensors with this dimension, although we have fabricated sensors with many width/length combinations.

\section{FREQUENCY RESPONSES}

The frequency responses of the sensors operating in constant current (CC) mode are presented here, as shown in Fig. 4. In this mode, the results do not depend on the parameters of the biasing circuit and are entirely determined by the structures and the material properties. Note that for type $I$, the frequency response is determined mainly by the diaphragm properties such as thickness and thermal conductivity. For type III, since the ends of the wire are directly connected to the substrate which behaves as a perfect heat sink, the frequency response is solely determined by the polysilicon wire. For type II and IV, the diaphragm and the support shanks to which the polysilicon wires are connected can heat up through thermal conduction; therefore, each device has two corner frequencies, one $(\sim 500 \mathrm{~Hz})$ corresponding to the diaphragm (or the

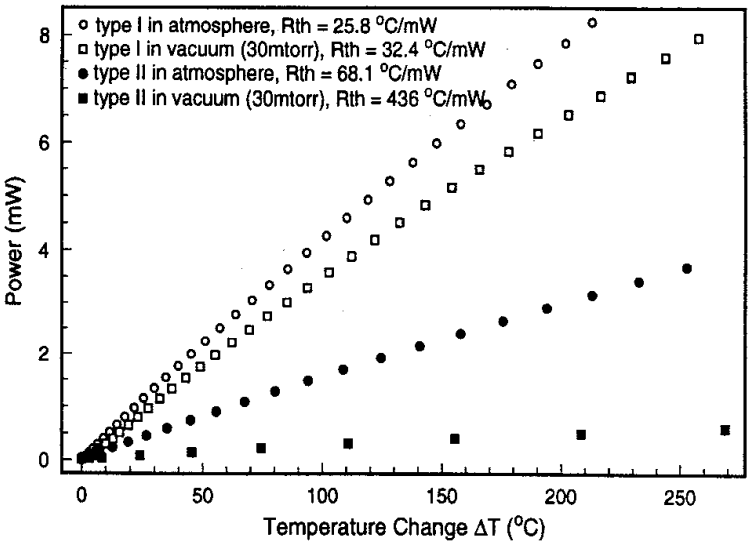

(a) Types I and II

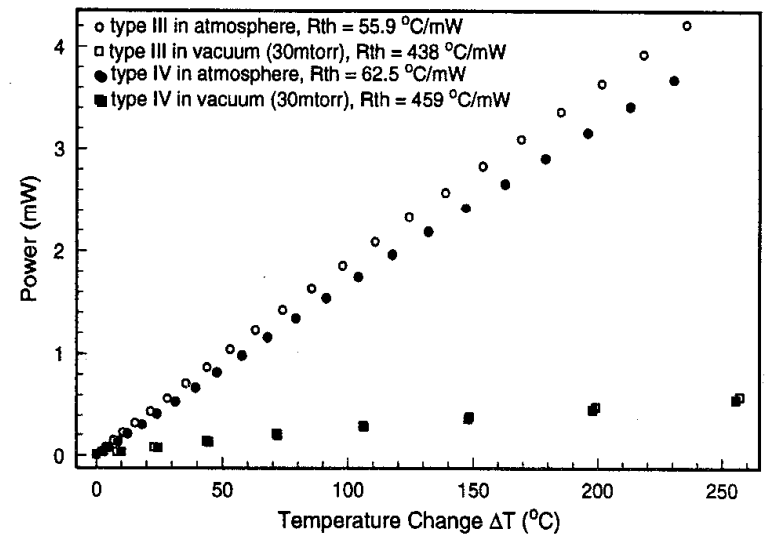

(b) Types III and IV

Fig. 5 Thermal resistances in atmosphere and in vacuum.

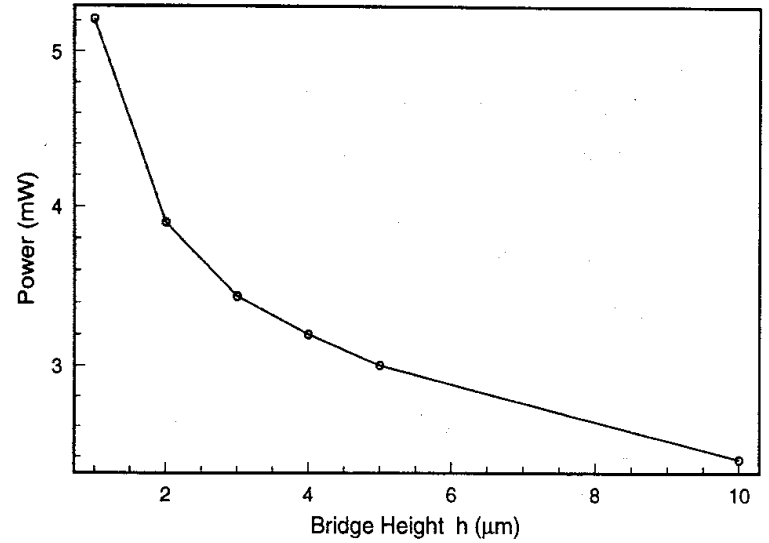

Fig. 6 The power needed to heat up type IV sensors with different air gap height.

support shanks) and the other $(3-4 \mathrm{kHz})$ to the wire. The effect of the second corner frequency can be greatly reduced if we increase the wire length or put aluminum over the support shanks to the ends of the wire.

It is worth mentioning that by operating them in constant temperature mode, all four types of sensors can reach a $-3 \mathrm{~dB}$ bandwidth over $10 \mathrm{kHz}$, at the expense of more complicated biasing circuitry, more noise and less stability. Therefore, in some cases, i.e., when arrays of sensors are required, the $\mathrm{CC}$ mode is preferred in order to simplify the interface electronics, enhance the stability and save power as long as the bandwidth meets the minimum requirement. Our study shows that in most wind-tunnels, the frequency bandwidth of the turbulence is around $1 \mathrm{kHz}$, which means that types II, III and IV are more useful in this application.

\section{HEAT TRANSFER ANALYSIS}

The heat dissipation of a heated element is a combination of thermal conduction, convection and radiation, although the first two mechanisms dominate for tempera-

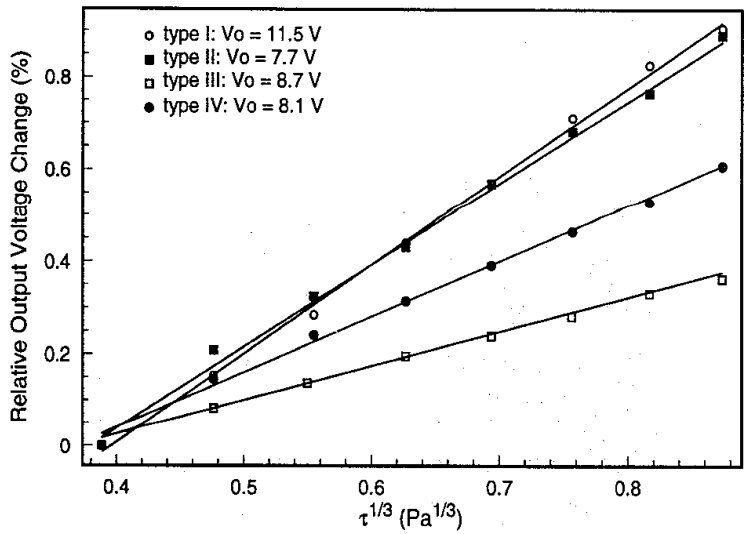

Fig. 7 Wind-tunnel responses in CT mode $\left(T=200^{\circ} \mathrm{C}\right)$. 
tures less than $300^{\circ} \mathrm{C}$. In order to separately study conduction and convection, we first measure the power needed to heat up the wire to a certain temperature in atmosphere. These samples are then mounted in a vacuum chamber ( 30 mtorr) to do the same measurements. Since convection and conduction to the substrate through the gap is negligible in such a low pressure, this heat is completely conducted away along the wire to the two ends. Fig. 5 shows the $\boldsymbol{P}$ (power) - $\Delta \boldsymbol{T}$ (temperature change) curves in atmosphere and in vacuum for each type of sensors. Here $\Delta T$ is the average wire temperature change and is calculated from the resistance change and the measured TCR (temperature coefficient of resistance) of $1 \times 10^{-3 /} \mathrm{C}$ for polysilicon. The reciprocals of the slopes of these curves represent the thermal resistances $\boldsymbol{R}_{t h}$. Type I has the smallest thermal resistance because the nitride diaphragm is also significantly heated. Types II - IV have nearly the same thermal resistances. Their slight differences reflect different end losses.

Another interesting test is to measure the power change as a function of the spacing distance, $\boldsymbol{h}$, between the wire and the substrate in atmosphere for types II - IV. This gives the information about the heat conduction loss to substrates through the air. As shown in Fig. 6, this loss can not be neglected for $\boldsymbol{h}$ less than $10 \mu \mathrm{m}$.

\section{WIND-TUNNEL EXPERIMENTS}

We used fully developed channel flow to do the calibration, with the sensors flush mounted on the wall. The shear stress can be calculated from the measured free stream mean velocity by using the following empirical formula for a fully developed turbulent channel flow [5]

$$
\tau=0.00826 R e^{-0.178} U_{\infty}^{2} \rho
$$

where $R e=\frac{U_{\infty} d}{y}$ is the Reynolds number, $d$ the half height of the wind-tunnel, $v$ the kinematic viscosity, $\rho$ the density of air and $U_{\infty}$ the free stream mean velocity. It can also be calculated from the measured streamwise pressure gradient by using

$$
\frac{\tau}{d}=-\frac{d P_{x}}{d x}
$$

Good agreement is found for shear stress values calculated using both methods.

Fig.7 shows the relative output voltage change vs. wall shear stress for all four types of sensors with air gap height of $4 \mu \mathrm{m}$ in constant temperature mode. In this mode, the square of the total output voltage $V^{2}$ should be proportional to the one third power of the wall shear stress $\tau^{1 / 3}$ [4]. Then if it is small, the relative output change is also proportional to $\tau^{1 / 3}$. It is obvious that our sensors follow this relationship closely. Types I and II are the most sensitive ones and have approximately the same relative sensitivity, despite the big difference in thermal resistances.
The reason might be that when the wire is not far above the diaphragm for type II, the interaction (heat transfer) between them is almost independent of the environment change and they can be considered as a single element, just as type I sensor. Also, both type I and type II have negligible conduction loss to the substrate and are certainly more sensitive than the other two types that have significant conduction loss to the substrate through the air gaps and/or through the ends (mainly for type III). Increasing the gap height reduces the conduction loss to the substrate and improves the sensitivity for types III and IV, but then it may interfere with the flow and make the sensor more vulnerable.

\section{CONCLUSION}

We have designed, fabricated and tested four polysilicon structures for wall shear stress sensors. Comparative studies have shown that they are all usable in flow study, with some trade-off between the frequency response and sensitivity.

\section{ACKNOWLEDGMENT}

This project is sponsored by the Air Force of Scientific Research, Air Force Material Command, USAF, under the grant number F49620-1-93-0332. The authors would like to thank Mr. Trevor Roper for the help with processing and Dr. Steve Tung for the help with wind-tunnel testing.

\section{REFERENCES}

[1] P. R. Bandyopadhyay, "Development of a Microfabricated Surface for turbulence Diagnostics and Control", Application of Microfabrication to Fluid Mechanics, Chicago, pp. 67-74, 1994.

[2] D. M. Bushnell, "Turbulent Drag Reduction for External flows", AIAA Papaer No. 83-0227, 1983.

[3] P. Moin, J. Kim, H. Choi, "On the Active Control of WallBounded Turbulent Flows", AIAA Paper No. 89-0960, 1989.

[4] R. J. Goldstein, Fluid Mechanics Measurements, Chapter 11, Hemisphere Publishing Corp., pp. 559-615, 1983.

[5] C. Liu, Y. C. Tai, J. B. Huang, C. M. Ho, "Surface Micromachined Thermal Shear Stress Sensor", Application of Microfabrication to Fluid Mechanics, Chicago, pp. 9-15, 1994.

[6] Y. C. Tai, R. S. Muller, "Lightly-Doped Polysilicon Bridge as Flow Meter", Sensors and Actuators, Vol. 15 (1), pp. 63-75, 1988.

[7] F. Jiang, Y. C. Tai, C. M. Ho, W. J. Li, "A Micromachined polysilicon Hot-Wire Anemometer", Tech. Digest 1994 SolidState Sensor and Actuator Workshop, Hilton Head, USA, pp. 264-267, 1994.

[8] F. Jiang, Y. C. Tai, C. M. Ho, R. Karan, M. Garstenauer, "Theoretical and Experimental Studies of the Micromachined Hot-Wire Anemometers", Tech. Digest 1994 IEDM, San Francisco, pp. 139-142, 1994.olid-State Sensor and Actuator Workshop, Hilton Head, USA, pp. 264-267, 1994. 